\title{
KALMAN PREDICTOR DESIGN FOR FREQUENCY-ADAPTIVE SCHEDULING OF FDD OFDMA UPLINKS
}

\author{
Daniel Aronsson and Mikael Sternad \\ Signals and Systems, Uppsala University, PO Box 534, SE-751 21 Uppsala, Sweden \\ \{daniel.aronsson,mikael.sternad\}@ signal.uu.se, tel. +46 18 4713078, fax +46 18555096
}

\begin{abstract}
Frequency-adaptive multiuser scheduling in OFDM utilizes the frequency-selective small-scale fading to allocate subcarriers with advantageous signal-to-noise ratio to each user. Due to channel time-variability and delays of the transmission control loop, this will in general require channel prediction. FDD (Frequency Division Duplex) uplinks pose the most challenging prediction problem: All sub-bands that may potentially be allocated must here be predicted for all involved user terminals, based on pilots transmitted from all terminals. This poses challenges with respect to prediction accuracy, estimator complexity and pilot overhead. This paper explores the design, performance and complexity of Kalman predictors used for uplink prediction, in the context of the EU WINNER project baseline design system. One conclusion is that uplink prediction that is useful at vehicular velocities in $4 \mathrm{G}$ systems operating at 3$5 \mathrm{GHz}$ is indeed feasible. However, the channel predictability depends crucially on the local fading environment, so predictors should be based on models of the Doppler spectrum for each terminal.
\end{abstract}

\section{INTRODUCTION}

The use of OFDMA (Orthogonal Frequency Division Multiple Access) on wide-band channels enables the allocation of users to the frequency regions that are most advantageous for them. A multiuser scheduler could allocate the transmission to/from each users to appropriate frequency bands, by utilizing the channel variations due to the small-scale frequency selective fading. Channels to/from each user will in general vary independently. Substantial multiuser scheduling gains can be attained if each of $K$ users on average is allocated to the fraction $1 / K$ of the subcarriers that will have highest SINR (signal to interference and noise ratio) for that particular user. Additional (but smaller) gains are obtained by using link adaptation that is adjusted to each allocated subcarrier.

Due to these potential gains, frequency-adaptive transmission in OFDMA downlinks is of interest in the research community, in the ongoing 3GPP long-term evolution (LTE) standardization effort [1], for WiMAX and in the European beyond-3G WINNER project [2]. One complication in such systems is that for moving terminals, measurements of the channel gain for a subcarrier will become quickly outdated. Fig. 1 illustrates the variation of received power with time and frequency for one particular user and fading pattern.

Frequency-adaptive transmission for vehicular users would therefore require low latency control loops for the transmission and also efficient channel prediction of the SINR at the instant of transmission. Designs for downlinks up to $100 \mathrm{~km} / \mathrm{h}$

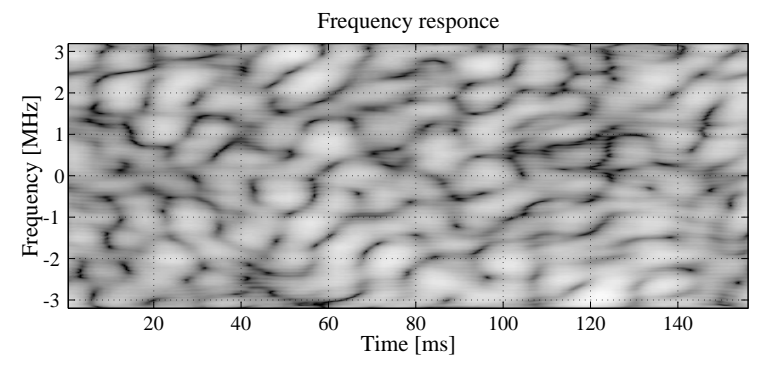

Figure 1: Time-frequency representation of an estimated channel obtained from measurement data on a $6.4 \mathrm{MHz}$ channel at a $1880 \mathrm{MHz}$ carrier. White color denotes high power whereas dark color denotes low power. The dynamic range and the speed of the mobile is approximately $40 \mathrm{~dB}$ and $50 \mathrm{~km} / \mathrm{h}$, respectively. The coherence bandwidth is $0.6 \mathrm{MHz}$ in this example.

at $2 \mathrm{GHz}$ carrier have been investigated within the Swedish Wireless IP project [3] and downlink designs targeted at up to $70 \mathrm{~km} / \mathrm{h}$ at $5 \mathrm{GHz}$ have been proposed and evaluated within the WINNER project $[4,5]$.

For FDD downlinks and TDD (time division duplex) uplinks and downlinks, channel prediction can be based on downlink pilots that are transmitted by the base station to all terminals within a sector or beam. ${ }^{1}$ Channel predictors at each terminal may then predict the frequency selective channel over a band of interest $[6,7]$. Suitably compressed messages are reported to the scheduler at the base station. The scheduler then allocates the use of each sub-band.

The potential multiuser scheduling gain is as large in FDD uplinks as in the cases outlined above, but the channel prediction problem becomes much harder: FDD uplinks cannot be predicted based on downlink pilots, because uplinks and downlinks work at significantly different carrier frequencies. Predictors at the base station would have to estimate the channels from all terminals who compete for a set of transmission resources. To support this prediction, all of these terminals would have to send special-purpose pilots within all resources of interest, at an appropriate channel sampling rate. ${ }^{2}$ The need for such special uplink pilots leads to two problems that become severe when the number of competing users is large:

1. If uplink pilots are transmitted in orthogonal positions,

\footnotetext{
${ }^{1}$ In TDD, prediction of the downlink channel gains can be used also for the uplink gains, due to the channel reciprocity.

${ }^{2}$ If only the pilots that are embedded in uplink payload transmissions are used, then the channel sampling would depend on the availability and scheduling of the uplink transmissions. Extrapolation to other frequency bands beyond the correlation bandwidth could not be performed. Also, the sampling in any given band could not be relied on to have sufficient rate to support a reliable prediction of the channel in that band.
} 
then the overhead of earmarked pilot positions relative to payload could become unacceptably large.

2. If $K$ users compete for a set of resources, each of them will on average obtain only $1 / K$ of the resources but will still have to transmit pilots in all of them. For large $K$, this pilot power overhead will negate the multiuser scheduling throughput gains.

The multiuser scheduling gains increase significantly with $K$ for small $K$ but slower for large $K$. This makes it possible to handle both of the problems outlined above by partitioning the total bandwidth into a number of competition bands, each with a limited number, typically $K=8$ or less, users. The competition bands should be composed of frequency resources that are well spread out over the uplink bandwidth, to sample the available frequency selectivity.

Our problem here will therefore be to design and assess channel predictors that work on uplink pilots that are transmitted from $K$ users, within a subset of OFDM subcarriers that constitute a competition band.

We will discuss various design aspects for Kalman-based schemes that produce MMSE estimates of the complex frequency-domain channel gains. Compared to Wiener filtering [8, 9], Kalman estimators provide better initial transient accuracy and are the optimal estimators for linear signal models and Gaussian noise. Kalman predictors also provide the prediction covariances. This uncertainty information can be used by the scheduler and by the link adaptation, to e.g. attain a target bit error rate at a given prediction variance [10].

The Kalman predictors can be implemented in the time domain, by tracking impulse response coefficients and then transforming them to predicted frequency domain channels. Alternatively, they can be implemented in the frequency domain. The performance of both these schemes has been evaluated, and is identical. We here show the results from the frequency domain implementation.

Initial results on Kalman-based predictors for FDD uplinks were reported in [11] and [4]. We will here discuss the effects of different pilot patterns and the channel fading statistics, which will each be illustrated for two extreme cases. The computational complexity, which determines the feasibility in fast adaptation feedback loops, will also be analyzed.

\section{System Model}

The required and the obtained prediction performance will be evaluated with respect to the baseline system design of the WINNER FDD mode [12]. This design has a system sampling period of $12.5 \mathrm{~ns}$, giving a FFT bandwidth of $80 \mathrm{MHz}$. The signal bands are $45 \mathrm{MHz}$ in both uplinks and downlinks. Each OFDM symbol is 2048 samples plus an additional 256 samples for the cyclic prefix. The subcarrier width is $39.06 \mathrm{kHz}$ and the OFDM symbol + guard duration is $28.8 \mu \mathrm{s}$.

The time-frequency radio resource is divided into blocks (chunks) of 8 subcarriers $(312.5 \mathrm{kHz})$ by 12 OFDM symbols $(345.6 \mu \mathrm{s})$. A chunk duration is denoted a slot. These chunks constitute the unit for frequency-adaptive resource allocation.
The chunk size is selected to make the channel moderately flat within chunks. Uplink pilot symbols known to the the receiver facilitate the prediction. They are here assumed located on one of the 12 OFDM symbols and no payload data is transmitted on this symbol. We here assume a full-duplex FDD uplink, so uplink pilots will be transmitted within each slot.

To prepare for frequency adaptive uplink transmission, the terminal is allocated a competition band and begins to send pilots in that band. Based on these pilots, an autoregressive model is adjusted to the temporal correlation (Doppler spectrum) and the frequency correlation of the fading is also estimated. These models are input data to the predictors and are used for assessing the attainable prediction accuracy.

Channel predictions are then produced for this users channel (see Section III below). When a packet for uplink transmission arrives, the terminal sends a transmission request during slot $j$. The scheduler may grant the request and sends the allocation information over a downlink control channel during slot $j+1$. The transmission then commences over the uplink in slot $j+$ 2. The required prediction horizon is two slots, or $0.7 \mathrm{~ms}$, or $L=2$ channel samples. This tight control loop requires the update of the channel prediction from the last measurement in slot $j$, the scheduling and the downlink control transmission to be executed within less than 1.5 slot durations $(0.5 \mathrm{~ms})$.

\section{STATE SPACE MODELLING}

We construct a linear filter that uses measurements of $W$ parallel pilot-bearing subcarriers. The fading taps of $U$ simultaneous users $^{3}$ are modeled by

$$
\begin{aligned}
x_{t+1} & =F x_{t}+G u_{t}, \\
h_{t} & =H x_{t} \\
\{F, G, H\} & =\operatorname{diag}_{\text {\#users }}\left(\operatorname{diag}_{\text {\#subc. }}\left(\left\{F_{s}, G_{s}, H_{s}\right\}\right)\right) .
\end{aligned}
$$

Here, $\operatorname{diag}_{a}$ is a block diagonal matrix holding $a$ blocks, and $h_{t}$ is a vector holding the $W \times U$ fading taps. Each triplet $\left\{F_{s}, G_{s}, H_{s}\right\}$ models the fading statistics of one tap with an autoregressive model of order 4 . The four poles of this model are so chosen as to represent a certain Doppler spectrum. The shape of the Doppler spectrum depends on the fading environment. When not explicitly stated otherwise, a flat Doppler spectrum in $\left[-f_{D}, f_{D}\right]$, where $f_{D}$ is the maximal Doppler frequency is used in this paper.

The vector $y_{t}$ of the $W$ received signals at the timefrequency pilot positions is modeled by placing the pilots of each user $j$ at time $t$ in diagonal $W \times W$ matrices $\left\{\psi_{j, t}\right\}$ and writing

$$
y_{t}=\operatorname{stack}_{\text {\#users }}\left(\psi_{j, t}\right) h_{t}+v_{t} .
$$

Here, $\operatorname{stack}_{a}$ is a matrix of $a$ blocks stacked horizontally, and $v_{t}$ represents noise and interference. Note that, unless we set most pilot symbols to zero, the received signal at a subcarrier will be affected by the channels from multiple users.

The correlation between the taps is expressed by the process noise covariance matrix $Q=\mathrm{E}\left[u_{t} u_{t}^{*}\right]$, while $R=\mathrm{E}\left[v_{t} v_{t}^{*}\right]$ is the noise covariance matrix. Here, we will assume $R=\sigma_{n}^{2} \mathrm{I}$.

\footnotetext{
${ }^{3}$ Channels for multiple transmit antennas/spatial streams from one terminal are here modelled as channels from different users.
} 


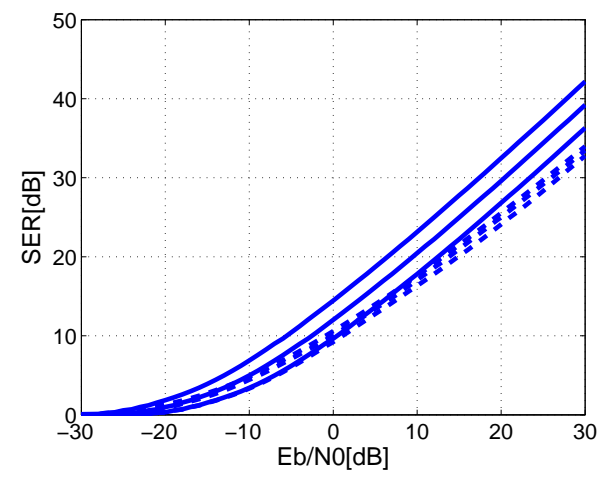

Figure 2: Filter performance versus filter width for widths 4,8 , and 16 . Solid lines show the performance on a flat fading channel. Dashed lines for the frequency selective WINNER C2 non-line-of sight channel.

With the state and measurement equations (1) and (2), optimal inferences about the taps $h$ are obtained by the Kalman equations. See e.g. [13]. The $L$-step prediction estimate of $h$ is obtained from the state estimate $\hat{x}_{t \mid t}$ by $\hat{h}_{t+L \mid t}=H F^{L} \hat{x}_{t \mid t}$.

The attainable prediction accuracy will depend on the prediction horizon $\ell$ scaled in carrier wavelengths, which in turn depends on the terminal velocity $v$, the prediction horizon in time $L t_{p}$ [s] and the carrier wavelength $\lambda_{c}$ via the relation $\ell=v L t_{p} / \lambda_{c}$. In the assumed WINNER baseline design, the horizon $L=2$, the sampling time $t_{p}$ equals the slot duration $(345.6 \mu \mathrm{s})$ and $\lambda_{c}=8.1 \mathrm{~cm}(3.7 \mathrm{GHz}$ uplink carrier).

\section{RESULTS}

\section{A. Channel model}

The results in this section are evaluated on two channel models: A flat (frequency non-selective) channel, and a frequency selective non-line-of sight channel for urban environments (WINNER C2 channel) with power delay profile

\begin{tabular}{|l|l|}
\hline \multicolumn{1}{|c|}{ Delay[ns] } & \multicolumn{1}{|c|}{ Power[dB] } \\
\hline $0,5,135,160,215,260$, & $-0.5,0.0,-3.4,-2.8,-4.6,-0.9$, \\
$385,400,530,540,650$, & $-6.7,-4.5,-9.0,-7.8,-7.4$, \\
$670,720,750,800,945$, & $-8.4,-11.0,-9.0,-5.1,-6.7$, \\
$1035,1185,1390,1470$ & $-12.1,-13.2,-13.7,-19.8$ \\
\hline
\end{tabular}

When not explicitly stated otherwise, we set the velocity of the terminals to $50 \mathrm{~km} / \mathrm{h}$, the average signal-to-noise ratio $E_{s} / N_{0}$ to $12 \mathrm{~dB}$, and the filter width $W$ to 8 subcarriers (one chunk width). The estimation horizon is set to two steps (slots). Performance is expressed either in terms of the mean value over all $W \times U$ channel taps of the signal-to-estimation error power ratio (SER), or by the normalized mean square error NMSE $=(\mathrm{SER})^{-1}$.

\section{B. Filter width}

For a filter tracking parallel subcarriers, an increased filter width $W$ should increase the performance. For a flat fading channel with noise without frequency correlation $\left(R=\sigma_{n}^{2} \mathrm{I}\right)$, the filter performance as measured by the signal-to-error ratio (SER) will increase by $3 \mathrm{~dB}$ when the filter width is doubled.

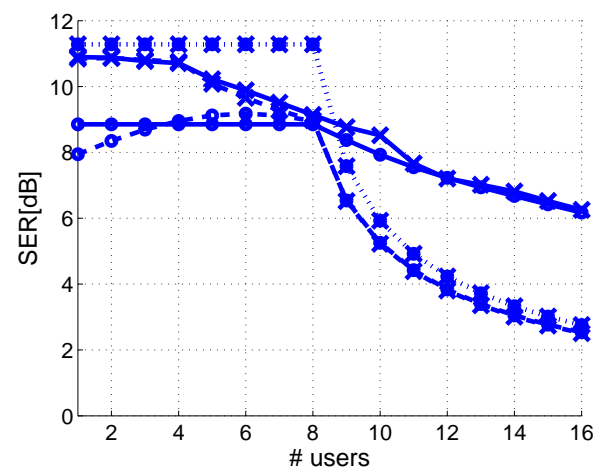

Figure 3: SER versus number of users on flat fading channels (dotted lines). Two-step prediction results for frequency selective channels with timeinvariant pilots (dashed lines), and frequency selective channels with cyclically time-varying pilots (solid lines). Rings indicate results for dedicated pilots, crosses indicate overlapping pilots. $W=8$, and average $E_{s} / N_{0}=12 \mathrm{~dB}$.

The improvement is not as pronounced when the channel is frequency selective. We illustrate this in Figure 2.

\section{Choice of pilots}

The choice of pilots is crucial to achieve a high performance. In this paper we evaluate two pilot schemes:

- Dedicated pilots, where each user puts pilots only on one subcarrier out of the $W$ subcarriers tracked by one filter, with zero energy on the subcarriers used by other users.

- The use of overlapped pilots, where all users place pilots on all $W$ subcarriers. We here use Walsh sequences to ensure that these pilots are orthogonal as long as the number of users $U$ is less than or equal to $W$.

When $U>W$ we can no longer find pilots that are orthogonal among all users. Pilots for users $W+1, W+2$ and so on are then constructed by pairwise combining pilots from the original set of $W$ orthogonal pilots.

The estimation and prediction performance will be improved by letting the pilots vary over time so that $\left[\operatorname{stack}_{U}\left(\psi_{j, t}\right)^{T} \ldots \operatorname{stack}_{U}\left(\psi_{j, t+W-1}\right)^{T}\right]^{T}$ has full rank $W U$. For time-invariant channels, we can then after $W$ steps obtain a linear system of equations that provides an exact solution for $U \leq W$ noise-free users. For dedicated pilots, such cyclic pilots are accomplished by shifting the pilot positions of all users cyclically. The overlapped pilot sequences are multiplied by Walsh sequences, creating a cycle of period $W$.

The result of using different pilot schemes, all using equal pilot transmit power, are summarized by Figure 3. It is evident that for the frequency selective channels, the use of overlapped pilots improves performance when $U<W$. This is because dedicated pilots are localized in frequency while the overlapping pilots sample all frequencies. For flat fading channels (dotted), both pilot schemes give identical results. The use of cycled pilots is crucial when $U>W$. The effects of the pilot patterns have been discussed in more detail in [14]. 


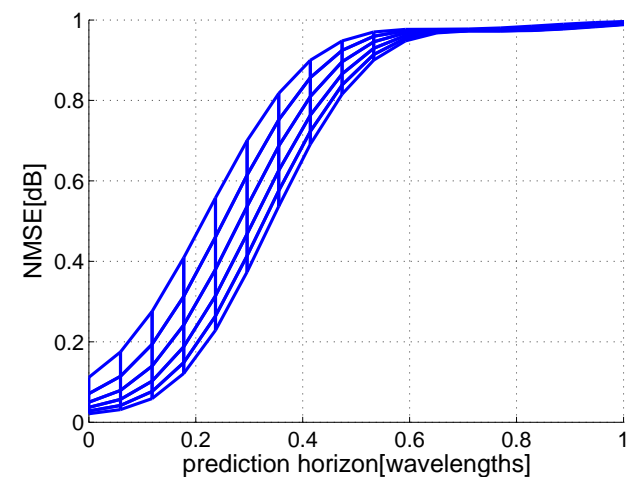

Figure 4: The predictor performance for flat Doppler spectrum measured by the NMSE, versus prediction horizon measured in wavelengths. The signal-tonoise ratio $E s / N_{0}$ goes from $0 \mathrm{~dB}$ (upper curve) to $25 \mathrm{~dB}$ (lower curve) in steps of $5 \mathrm{~dB}$. $U=8$ users with overlapping pilots, $W=8$, WINNER C2 channel model.

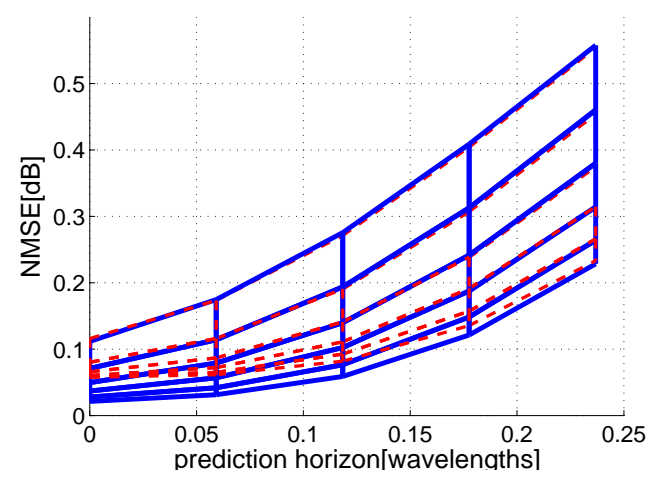

Figure 5: Detail study of the graph in Figure 4. Here we also add the prediction performance when time-invariant pilots are used (dashed lines), as opposed to cyclically varying pilot patterns (solid lines).

\section{Prediction horizon}

Here we evaluate the prediction performance for different signal-to-noise ratios $\left(E s / N_{0}\right)$ over a wide range of prediction horizons. The result for a flat Doppler spectrum is presented in Figure 4. Overlapped pilots that vary cyclically in time were used. When the prediction NMSE is below 0.15, frequencyadaptive multi-user scheduling can provide performance gains $[4,5]$. $¿$ From the expression $\ell=v L t_{p} / \lambda_{c}$ in Section III, the required prediction horizon is, for example, $\ell=0.12$ when $v=50 \mathrm{~km} / \mathrm{h}$ and $L=2$. The minimal acceptable prediction performance would then be attained when $E_{s} / N_{0}=10 \mathrm{~dB}$.

In Figure 5, we illustrate the impact of using cyclic pilots. Use of time-invariant pilots results in an error floor for short horizons and high SNR:s, since they provide less information about the past time-frequency variability of each channel.

\section{E. The impact of the local scattering environment}

The Doppler spectrum, caused by the angular distribution of local scatterers around each terminal, relative to its direction of travel, has a crucial impact on the channel predictability. So

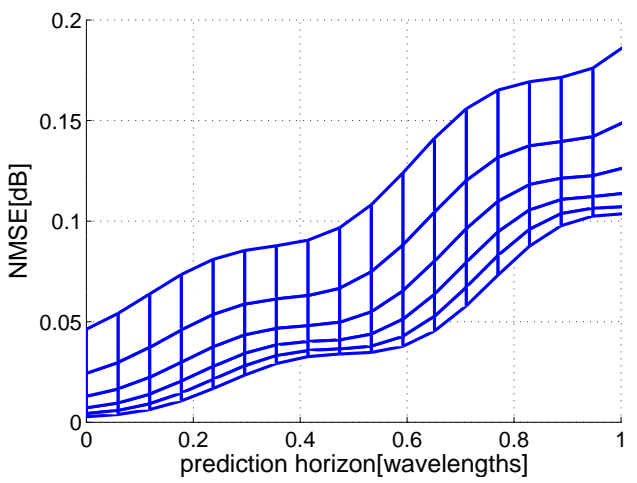

Figure 6: The predictor performance for oscillative AR4 Doppler spectrum measured in NMSE, versus prediction horizon measured in wavelengths. The signal-to-noise ratio $E s / N_{0}$ goes from $0 \mathrm{~dB}$ (upper curve) to $25 \mathrm{~dB}$ (lower curve) in steps of $5 \mathrm{~dB}$. $U=8$ users with overlapping pilots. $W=8$, WINNER C2 channel model.

far we have used a fading model with Doppler spectrum that is almost flat for frequencies less than the maximum Doppler frequency $f_{D}$. This corresponds to a situation where scatterers are placed mainly sideways relative to the direction of travel, e.g. due to buildings along streets.

An opposite extreme case is illustrated for the same situation as in Figure 4, but for a Doppler spectrum modelled by 4 poles close to the unit circle. This corresponds to a situation dominated by reflectors in front of or behind the terminal. This results in very good predictability, see Figure 6 . For all $E s / N_{0}$-levels, the prediction NMSEs stay below the limit 0.15 all the way up to a horizon of about 0.7 wavelengths.

\section{F. Numerical complexity}

To evaluate the feasibility of using optimal filters as proposed in this paper, we need to assess the complexity of the Kalman filter (KF). The complexity is mainly determined by the number of states $n$, which is the product of the fading model order $K$, the number of modelled taps $X$, and the number of users $U$. The value of $X$ depends on how the modelling is carried out: If time-domain prediction of $M$ taps is used, we set $X=M$. Otherwise we use frequency-domain prediction and choose $X=W$. Both cases are covered below.

The KF has to produce channel tap estimates (update the state estimates $\hat{x}$ ), predictions of the channel taps, as well as updates and predictions of the state error covariance matrix $P$. Due to the block diagonal structures used in this paper, the complexity is reduced considerably as compared to the general KF. It can be shown (see [15]) that the number of complex operations required for one $\mathrm{KF}$ update is

$$
\begin{array}{ll}
(3 / 2+W / 2) n^{2}+\left(K W+W^{2}\right) n & P \text { update } \\
\quad+K W^{2} / 2+W^{3} / 6 & \\
3 n^{2} / 2 & P \text { prediction } \\
(W+1) n+K W & \hat{x} \text { update } \\
n & \hat{x} \text { prediction } \\
\hline
\end{array}
$$

To cover a competition band that contains $C$ predicted subcarriers, int $[C / W] \mathrm{KFs}$ are run in parallel. The solid lines in 


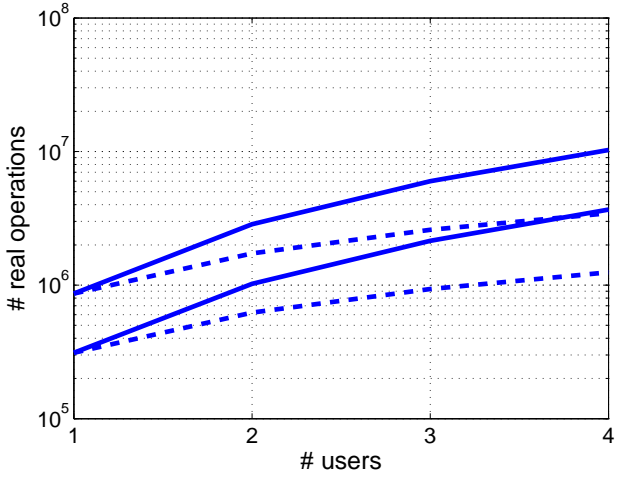

Figure 7: Total numerical complexity vs. number of users $K$ for predicting a competition band containing 160 predicted subcarriers, using either $40 \mathrm{KFs}$ of filter width $W=4$ (lower) or $20 \mathrm{KFs}$ with width $W=8$ (upper). Solid lines represent a general choice of pilots. Dashed lines represent the use of dedicated pilots and $K$ decoupled KFs for each set of $W$ subcarriers.

Figure 7 display the number of real operations required per update versus number of users for $C=160$ predicted subcarriers and $K=4$ uplink users per competition band, for designs with $W=4$ or $W=8$. An operation represents mult + add. Furthermore we assume that one complex operation has the same complexity as four real operations.

The above calculations hold for general choices of pilot symbols, but the complexity may be decreased further by considering only dedicated pilots (as opposed to overlapping pilots). The measurement equation is then completely decoupled between different users, which makes the state error covariance matrix $P$ block diagonal. This, in turn, means that we may run a separate KF for each user without losing performance, which means that the complexity increases only linearly with the number of users. In that case, the complexity is easily computed by setting $n=K X$ instead of $n=K X U$ in the above expressions, and then multiplying the final result by $U$. The dashed lines of Figure 7 show the number of real operations required for one update for filter widths $W=4$ and $W=8$ when these decoupled KFs can be used.

The WINNER baseline system would require a new prediction for each slot of duration $0.34 \mathrm{~ms}$ for vehicular users. To assess the feasibility of the required computational complexity, we here investigate the consequences of setting $10^{10}$ real operations per second as a target for feasibility for uplink predictors realized in the base station. ${ }^{4}$ This would correspond to a limit to $3.4 \cdot 10^{6}$ operations per update $(0.345 \mathrm{~ms})$. As is evident from Figure 7 (solid), using eight parallel subcarriers would then be infeasible, while four parallel subcarriers touches upon the limit. For the decoupled case (dashed), the total complexity of filters of width 4 falls well within our boundary while $W=8$ is on the limit for $k=4$. The use of Kalman-based uplink prediction seems feasible under these assumptions.

The numerical load imposed by the KF is dominated by the covariance matrix update. However, $P$ usually converges very quickly to a stationary value (the solution to the Discrete Alge-

\footnotetext{
${ }^{4}$ Lower targets would be realistic for predictors located in terminals.
}

braic Riccati Equation) when the model matrices are kept constant. This holds also when cyclic pilots are used, in the sense that $P$ will then approach a cyclostationary state in which the same value reoccurs with the same period as the cyclic pilots. In the experiment performed in this paper, $P$ converged to a fixed value ${ }^{5}$ in a few tens of iterations, and for most cases the iteration count was below 20 .

Therefore, Kalman iterations only need to be performed burst-wise when fading models or number of users change, due to the fast convergence of $P$. This reduces energy consumption relative to the case of continuous updates.

\section{REFERENCES}

[1] H. Ekström, A. Furuskär, J. Karlsson, M. Meyer, S. Parkvall, J. Torsner and M. Wahlquist, "Technical solutions for the 3G long-term evolution," IEEE Commun. Magazine, pp. 38-45, March 2006.

[2] IST WINNER and WINNER II projects, partly funded by the European Commission. Online: https://ist-winner.org ${ }^{6}$

[3] M. Sternad, T. Ottosson, A. Ahlén and A. Svensson, "Attaining both coverage and high spectral efficiency with adaptive OFDM downlinks," VTC 2003-Fall, Orlando, Fla, Oct. 2003.

[4] IST-2003-507581 WINNER, "D2.4: Assessment of Adaptive Transmission Technologies," Feb. 2005. Available on https://ist-winner.org.

[5] M. Sternad, S. Falahati, T. Svensson and D. Aronsson, "Adaptive TDMA/OFDMA for wide-area coverage and vehicular velocities," IST Mobile and Vehicular Summit, Dresden, June 19-23 2005.

[6] T. Ekman Prediction of Mobile Radio Channels. Modeling and Design PhD Thesis, Signals and Systems, Uppsala University, Sweden, 2002. Online: www.signal.uu.se/Publications/abstracts/a023.html

[7] M. Sternad and D. Aronsson, "Channel estimation and prediction for adaptive OFDM downlinks," IEEE VTC 2003-Fall, Orlando, Fla, Oct. 2003.

[8] P. Hoeher, S. Kaiser and P. Robertson, "Two-dimensional pilot-symbolaided channel estimation by Wiener filtering," IEEE ICASSP, 1997, pp. 1845-1848

[9] G. Auer, "Analysis of pilot-symbol aided channel estimation for OFDM systems with multiple transmit antennas," IEEE Intern. Conf. on Commun. ICC 04, Paris, June 2004.

[10] S. Falahati, A. Svensson, M. Sternad and T. Ekman, "Adaptive modulation systems for predicted wireless channels," IEEE Trans on Commun., vol. 52, pp. 307-315, Feb. 2004.

[11] M. Sternad and D. Aronsson, "Channel estimation and prediction for adaptive OFDMA/TDMA uplinks based on overlapping pilots," IEEE ICASSP, Philadelphia, March 19-23 2005.

[12] IST-4-027756 WINNER II, "D6.13.7: Test Scenarios and Calibration Cases; Issue 2," Dec. 2006. Available on https://ist-winner.org.

[13] T. Kailath, A.H. Sayed and B. Hassibi, Linear Estimation Prentice-Hall, Upper Saddle River, NJ, 2000.

[14] D. Aronsson and M. Sternad "OFDMA uplink channel prediction to enable frequency-adaptive multiuser scheduling," EUSIPCO, Poznan, Poland, September 2007.

[15] D. Aronsson, "Channel estimation and prediction from a Bayesian perspective,” Licenciate Thesis, Signals and Systems, Uppsala U. 2007.

\footnotetext{
${ }^{5}$ We consider $P$ to have converged when the maximum element-wise relative change in magnitude between iterations is below one percent.

${ }^{6}$ This work has been partially performed within the project IST-3-027756 WINNER II, which is partially funded by the European Union. The authors wish to acknowledge the contributions of their colleagues. It was also partially funded by the Swedish Foundation of Strategic Research.
} 